\title{
Uso do parafuso transfacetário para estabilização da coluna cervical: nota técnica
}

\author{
Ricardo Vieira Botelho', Edson Denner Zandonadi Ferreira², Helton Portela Santos \\ Bezerra², Rodolfo de Moura Carneiro², Emilio Afonso França Fontoura ${ }^{3}$ \\ Conjunto Hospitalar do Mandaqui
}

\section{RESUMO}

A utilização do parafuso para fixação da coluna cervical substituiu as técnicas de estabilização com amarrilhas sublaminares, que agora estão destinadas a raros casos em que há impossibilidade de ancoramento ósseo vertebral. Os sistemas de fixação com parafusos ganharam uso disseminado. As técnicas de fixação na massa lateral cervical, no pedículo e na lâmina tiveram seu uso disseminado. Embora a descrição da fixação com parafuso transfacetário cervical date da década de 1970, há muito pouca quantidade de literatura relacionada à técnica. Os autores descrevem a técnica de colocação do parafuso transarticular, ilustrando com a descrição do tratamento de um paciente vítima de fratura-luxação com bloqueio facetário, estabilizado pelo método.

\section{ABSTRACT}

The role of transfacetary screw in cervical spine surgery: technical note

Since the first description of cervical spine screw fixation, techniques using sublaminar wire fixation are intended to the rare cases with non-vertebral bone anchorage. Screw fixation systems has gained widespread use in recent times. The techniques of lateral mass fixation, cervical pedicle and the laminar screw had, in the same way, widespread use. Although the description of cervical transfacet screw fixation date of the 70s, there is very little amount of literature related to the technique. The authors describe the technique of transarticular screw placement illustrating the treatment of a patient victim of fracture dislocation with facet block, stabilized by the method.

\section{Introdução}

A fixação posterior da coluna cervical evoluiu das amarrilhas sublaminares para os sistemas de ancoramento ósseo com parafusos. Várias técnicas de fixação posterior na coluna cervical foram desenvolvidas. ${ }^{1}$ A fixação na massa lateral foi descrita por Roy-Camille. ${ }^{7}$

$\mathrm{O}$ uso do parafuso pedicular cervical e do parafuso laminar, principalmente em C2 e C7, tem sido descrito. ${ }^{3}$

Os parafusos na massa lateral cervical ganharam maior popularidade entre os cirurgiões, mas, mesmo eles, necessitam de estrito seguimento de orientação na colocação para evitar lesão radicular e da artéria vertebral por violação dos forâmens dessas estruturas. ${ }^{7}$

Os parafusos pediculares, embora biomecanicamente mais resistentes, não se provaram superiores clinicamente aos parafusos na massa lateral. ${ }^{4}$

Será descrita a técnica de colocação do parafuso transfacetário na coluna cervical ilustrando com um caso clínico.

\section{Relato do caso}

Paciente do gênero masculino, 22 anos, foi vítima de acidente motociclístico e atendido no pronto-socorro de outro hospital. $\mathrm{O}$ exame neurológico era normal e o exame radiográfico inicial da coluna cervical (Figura 1) mostrava fratura-luxação cervical com bloqueio facetário entre C6 e C7. Com o diagnóstico de fratura-luxação, foi encaminhado ao nosso hospital. A radiografia de controle e a ressonância magnética da coluna mostraram redução espontânea do bloqueio facetário e da subluxação (Figuras 2 e 3, à esquerda).

O socorrista do pronto-socorro considerou o caso estável, indicou colar cervical tipo Filadélfia e o encaminhou ao ambulatório. Na avaliação de ambulatório, o paciente foi submetido à radiografia em flexão e extensão acompanhada (Figura 3). As facetas articulares de C6 e C7 revelam subluxação durante a flexão, com angulação vertebral maior que $11^{\circ}$ (Figura 3 central; flexão).

1. Médico neurocirurgião, doutor e pós-doutor em Ciências pela Universidade Federal de São Paulo (Unifesp).

2. Médico neurocirurgião.

3. Médico neurocirurgião, diretor de Serviço de Neurocirurgia, mestre em Neurocirurgia pela Unifesp. 


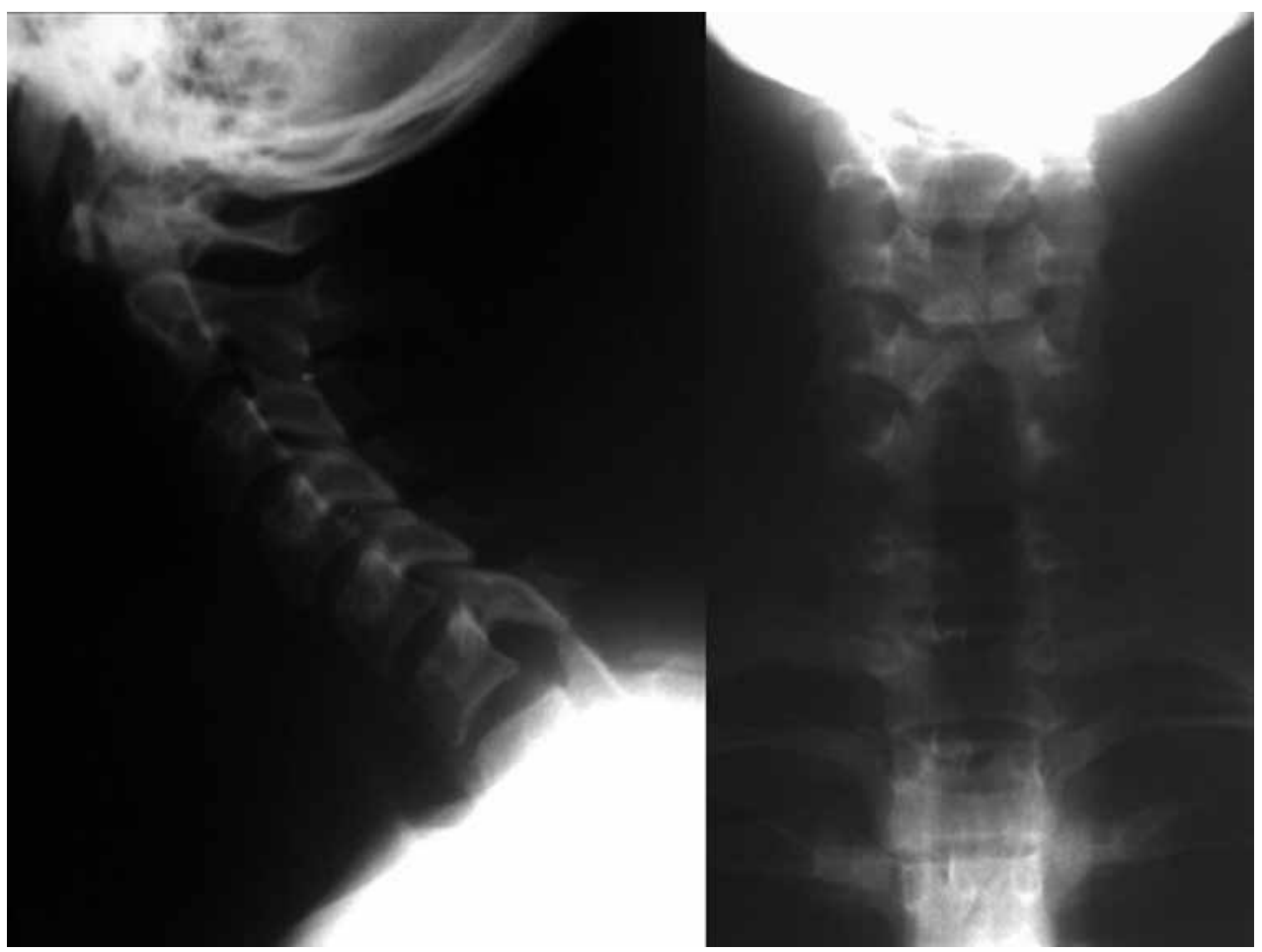

Figura 1 - Fratura-subluxação cervical. À esquerda, nota-se o bloqueio das articulações facetárias entre C6 e C7. À direita, há alargamento do espaço enterespinhoso entre $\mathrm{C6}$ e $\mathrm{C7}$, com rotação dos processos espinhosos.

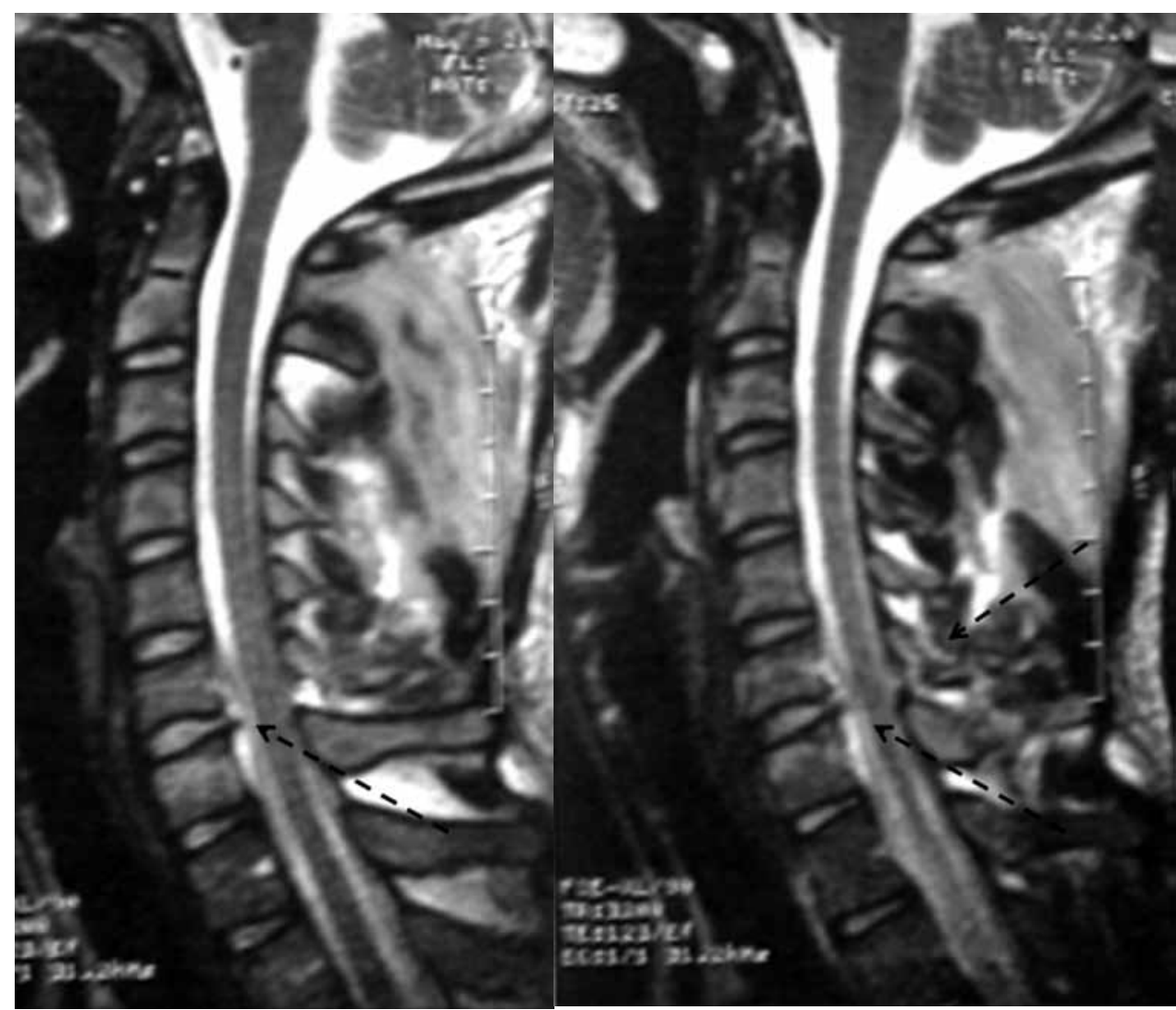

Figura 2 - Ressonância magnética mostrando a redução da subluxação. Na imagem da esquerda, a seta aponta para hiperintensidade na projeção do ligamento longitudinal anterior (LLA). Na imagem da direita, as setas apontam para a lesão do LLA (seta à esquerda) e para a lesão ligamentar posterior associada à fratura do processo espinhoso (seta à direita). 


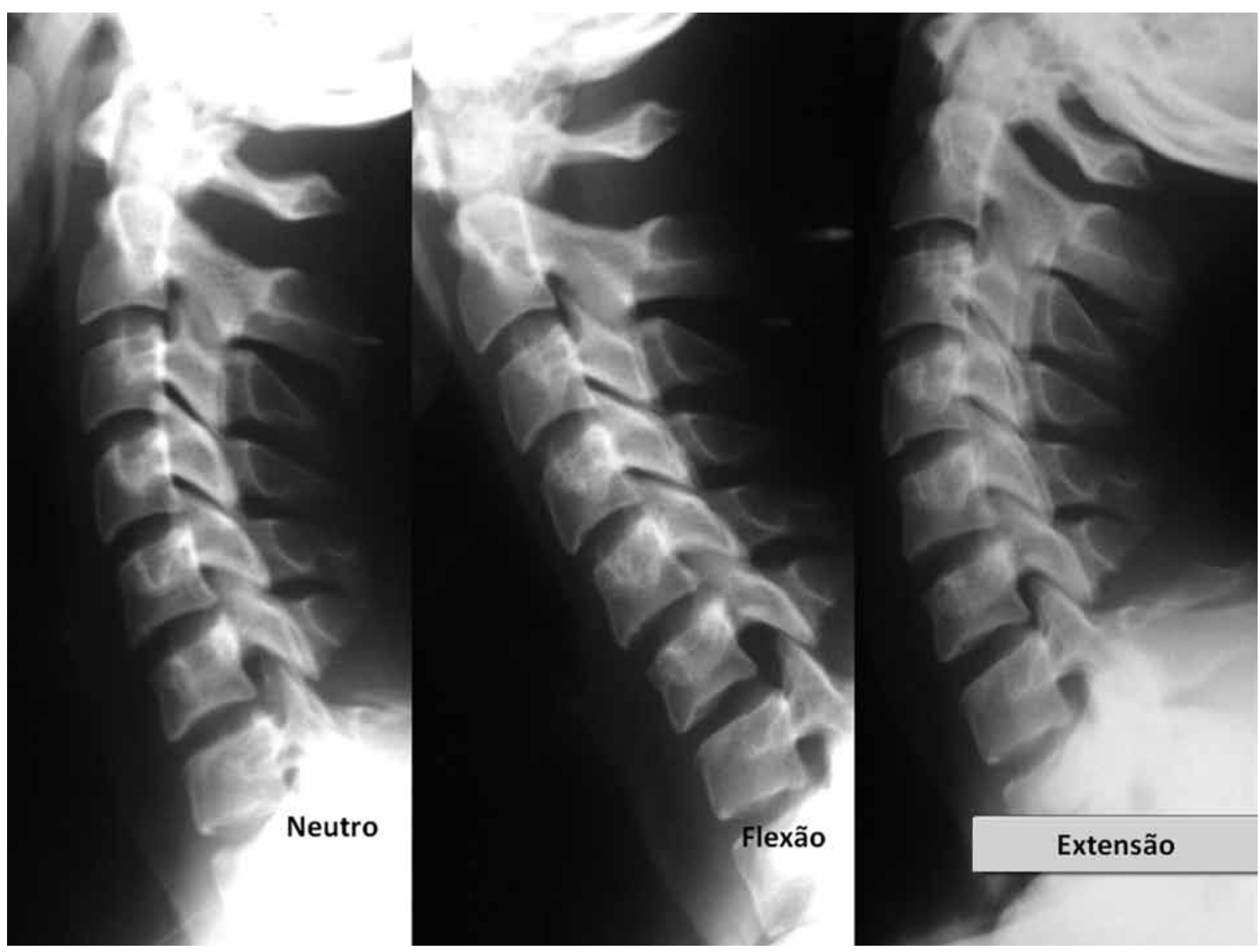

Figura 3 - À esquerda, nota-se a redução da subluxação (neutro). No centro, ocorre subluxação facetária durante a flexão. À direita, há redução da subluxação na extensão.

Em virtude da carga antecipada estimada alta para a coluna cervical em paciente jovem, ${ }^{10}$ este foi submetido à cirurgia para estabilização.

\section{Descrição da técnica}

O paciente foi colocado em posição prona, em decúbito ventral, com a cabeça apoiada pelo suporte de Mayfield radiotransparente (Figura 4), sob anestesia geral. Foram feitas duas incisões paravertebrais em 2,5 cm de extensão de cada lado da linha média (Figura 4), guiadas pela projeção das facetas articulares de C6 e C7 na radioscopia. A dissecção se faz através dos planos musculares até o plano das facetas (massas laterais). A abordagem pela linha média pode ser feita. ${ }^{9}$ As massas são identificadas e a faceta articular inferior de C6 é isolada para identificação de quatro quadrantes facetários. ${ }^{6} \mathrm{O}$ ponto de perfuração inicial se localiza $1 \mathrm{~mm}$ medial ao centro da massa lateral (Figura 5). A perfuração, no plano coronal, é direcionada do ponto inicial ao processo transverso da vértebra inferior, e no plano sagital a perfuração é guiada pela radioscopia, com o objetivo de parar o parafuso imediatamente após a perfuração da cortical interna da faceta superior de C7, preservando-se de qualquer penetração no forame e lesão radicular (Figuras 5 e 6). O parafuso utilizado foi o parafuso cortical, componente do sistema de fixação anterior da coluna cervical com placa e parafuso (Slim-LOC ${ }^{\circledR}$ Anterior Cervical Plate System-Depuy).

\section{Discussão}

A técnica da fixação transfacetária na coluna cervical média não tem sido frequentemente publicada nem tem ganhado popularidade. Provavelmente, isso se deve à grande popularidade da fixação na massa lateral cervical. ${ }^{2}$ 


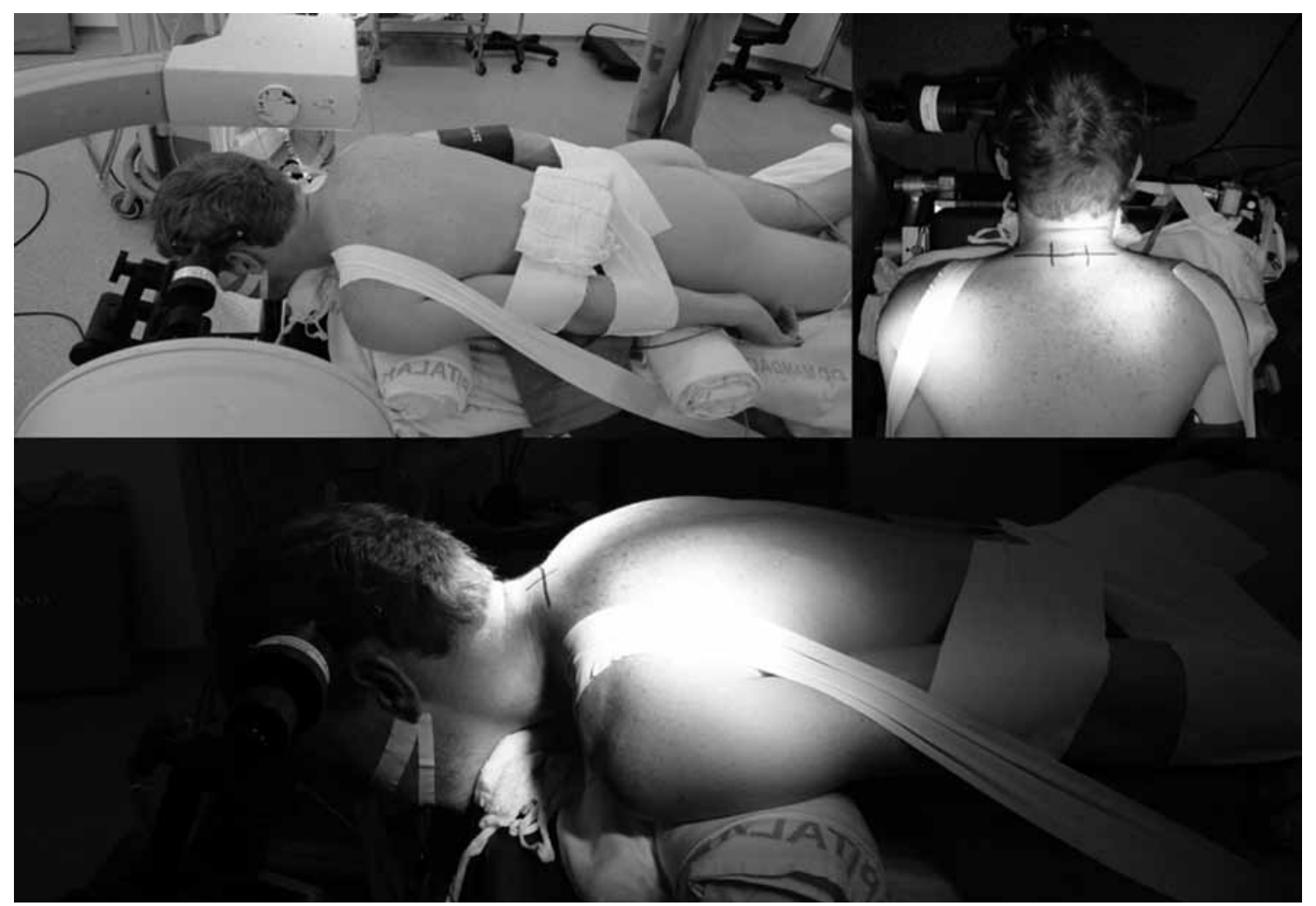

Figura 4 - Posicionamento durante a cirurgia. Na figura superior direita, estão representadas as duas incisões paramedianas utilizadas.

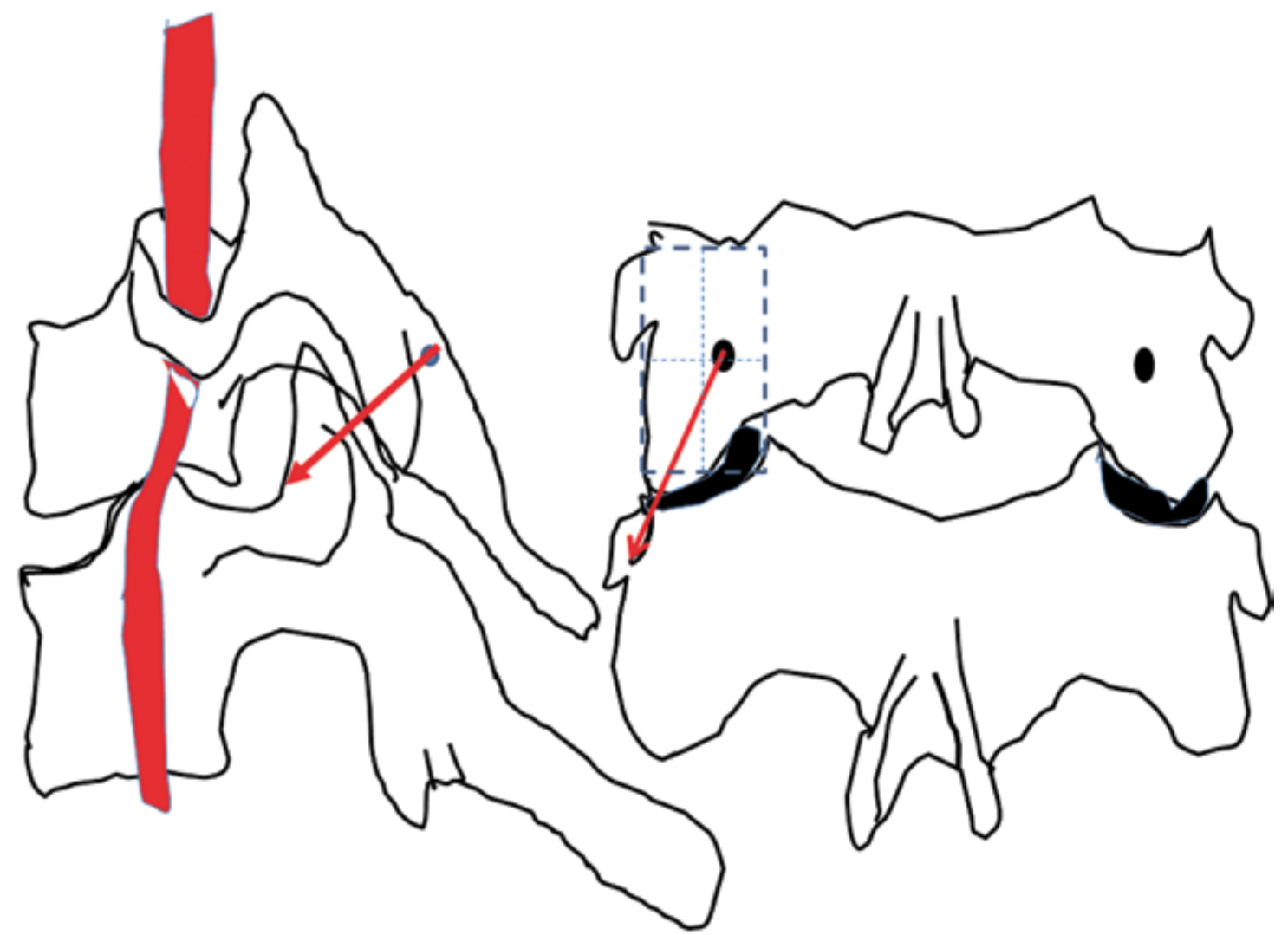

Figura 5 - Representação esquemática da colocação do parafuso. À esquerda, o trajeto é representado do meio da massa lateral superior, dirigindo-se para a cortical anterior da faceta superior da vértebra abaixo. À direita, mostra-se a projeção inferior oblíqua do parafuso, dirigindo-se para o processo transverso da vértebra de baixo. 


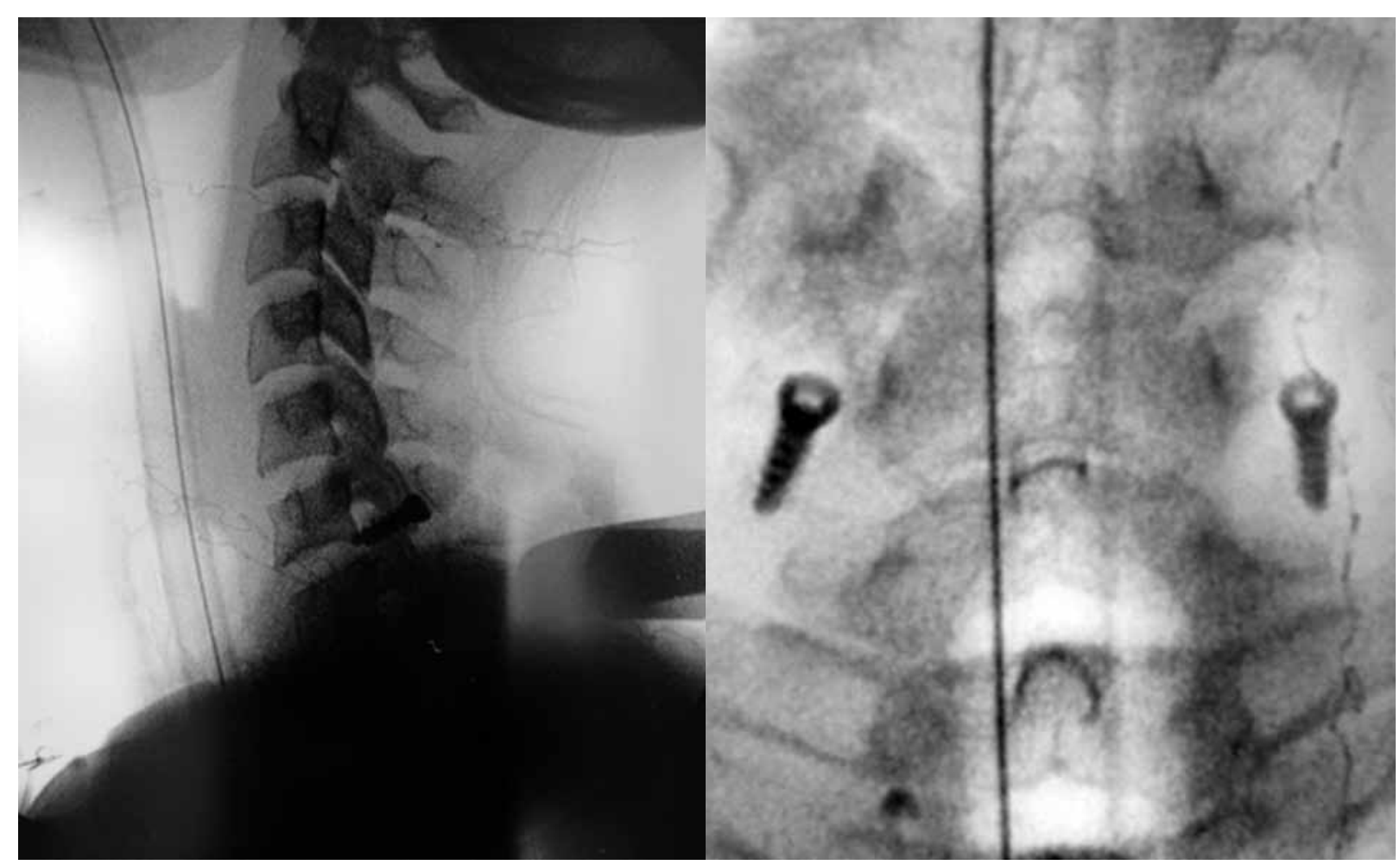

Figura 6 - Aspecto radiográfico no perfil (esquerda) e PA (direita) do parafuso transarticular.

É uma técnica simples ${ }^{9}$ e, nas fixações monossegmentares (como no caso do paciente descrito), pode ser feita com pequenas incisões, de acordo com o modelo de cirurgia minimamente invasiva, evitando o descolamento subperiostal da musculatura.

Uma vantagem imediata é o menor custo, pois utiliza apenas dois parafusos dos sistemas de fixação anterior. A orientação de colocação dos parafusos com trajeto limitado intraósseo aumenta a segurança da técnica em relação aos parafusos das massas laterais que utilizam parâmetros anatômicos para colocação. ${ }^{6,8}$

Os parafusos transfacetários cervicais apresentam resistência comparável à dos parafusos nas massas laterais cervicais. ${ }^{4,5}$

\section{Conclusão}

A técnica do parafuso transfacetário cervical é simples e eficiente em fixações da coluna cervical média.

\section{Referências}

1. Horn EM, Reyes PM, Baek S, Senoglu M, Theodore N, Sonntag VK, et al. Biomechanics of C-7 transfacet screw fixation. J Neurosurg Spine. 2009;11(3):338-43.

2. Hong JT, Tomoyuki T, Udayakumar R, Espinoza Orías AA, Inoue N, An HS. Biomechanical comparison of three different types of $\mathrm{C} 7$ fixation techniques: lateral mass screw, transpedicular screw, and intralaminar screw. Spine. 2011;36(5):393-8.

3. Jang SH, Hong JT, Kim IS, Yeo IS, Son BC, Lee SW. C7 posterior fixation using intralaminar screws: early clinical and radiographic outcome. J Korean Neurosurg Soc. 2010;48(2):129-33.

4. Jones EL, Heller JG, Silcox DH, Hutton WC. Cervical pedicle screws versus lateral mass screws: anatomic feasibility and biomechanical comparison. Spine. 1997;22(9):977-82.

5. Klekamp JW, Ugbo JL, Heller JG, Hutton WC. Cervical transfacet versus lateral mass screws: a biomechanical comparison. J Spinal Disord. 2000;13:515-8.

6. Pait TG, McAllister PV, Kaufman HH. Quadrant anatomy of the articular pillars (lateral cervical mass) of the cervical spine. J Neurosurg. 1995;82(6):1011-4.

7. Roy-Camille R, Saillant G, Laville C, Benazet JP. Treatment of lower cervical spinal injuries: C3 to C7. Spine. 1992;17:S442-6. 
8. Solanki GA, Crockard HA. Peroperative determination of safe superior transarticular screw trajectory through the lateral mass. Spine. 1999;24(14):1477-82.

9. Takayasu M, Hara M, Yamauchi K, Yoshida M, Yoshida J. Transarticular screw fixation in the middle and lower cervical spine: technical note. J Neurosurg. 2003;99(1 Suppl):132-6.

10. White 3rd AA, Johnson RM, Panjabi MM, Southwick WO. Biomechanical analysis of clinical stability in the cervical spine. Clin Orthop Relat Res. 1975;109:85-96.
Endereço para correspondência

Ricardo Vieira Botelho

Av. Dr. Altino Arantes, 390, ap. 81

04042-000 - São Paulo, SP

E-mail: vieira.botelho@uol.com.br

Telefax: (11) 5088-8379 